\title{
Effects of size and structure of xenogenic bone particles on foreign body reaction as a pattern of successful oseointegration
}

Pejovic Marko*, Dragovic Miroslav*, Stepic Jelena *, Milosevic Natasa*, Colic Snjezana* / Pejovic Marko

* Faculty of Dental Medicine, University of Belgrade, Serbia

\section{Background and Aim}

All biomaterials, when implanted in vivo, in the first couple of weeks induce foreign body reacion which may considerably have an impact on short and long-term success of biomaterial integration, necessiting a clear understanding of foreign body reaction to different bone substitutes.

The main objective of the research was to evaluate whether size and structure of bone substitute particles have an effect on presence and intensity of foreign body reaction.

\section{Methods and Materials}

Experimental study was carried out on rabbit animal model. Study included ten New Zealand rabbits. On both sides of cranial vault two 8$\mathrm{mm}$-diameter defects were formed which were filled with bone substitute particles and covered with collagen membrane. The tested materials are commercially available in two dimensions and included: deproteinized bovine bone minerals in the form of small and large particles (Bio-Oss $250-1000 \mu \mathrm{m}$ and $1000-2000 \mu \mathrm{m}$, respectively), and deantigenated equine-derived bone in the form of small and large particles (Osteoxenon $500-1000 \mu \mathrm{m}$ and $2000-3000 \mu \mathrm{m}$, respectively). After 4 and 8 weeks the animals were sacrifised, and tissue samples were prepared for pathohistological analysis. The bone particles integration were analized using histological, histochemical and histomorphometrical methods.

The following parameters were quantitatively evaluated:

1) foreign body giant cells number per $\mathrm{mm}^{2}\left(1 \mathrm{~mm}^{2}\right.$ corresponds to 8 fields of large magnification, 200x

2) blood vessels number per $\mathrm{mm}^{2}$

3) the highest width of fibrovascular strips (in $\mu \mathrm{m}$ )

4) the highest width of bone trabecula

\section{Results}

The number of foreign body giant cells (FBGCs) was significantly higher in case of small particles after 4 and 8 weeks of healing regardless the type of xenogenic origin. Furthermore, in defects filled with small particles significant reduction in number of FBGCs was noticed after 8 weeks of healing. Considering large particles, in case of Osteoxenon defects the number of FBGCs after 4 weeks was negligibly small and so is left after 8 weeks. The fact that FBGCs are procatabolic ones impeding new bone formation indicate that limited rate of new bone production which was measured in case of defects filled with small particles Fig.6).

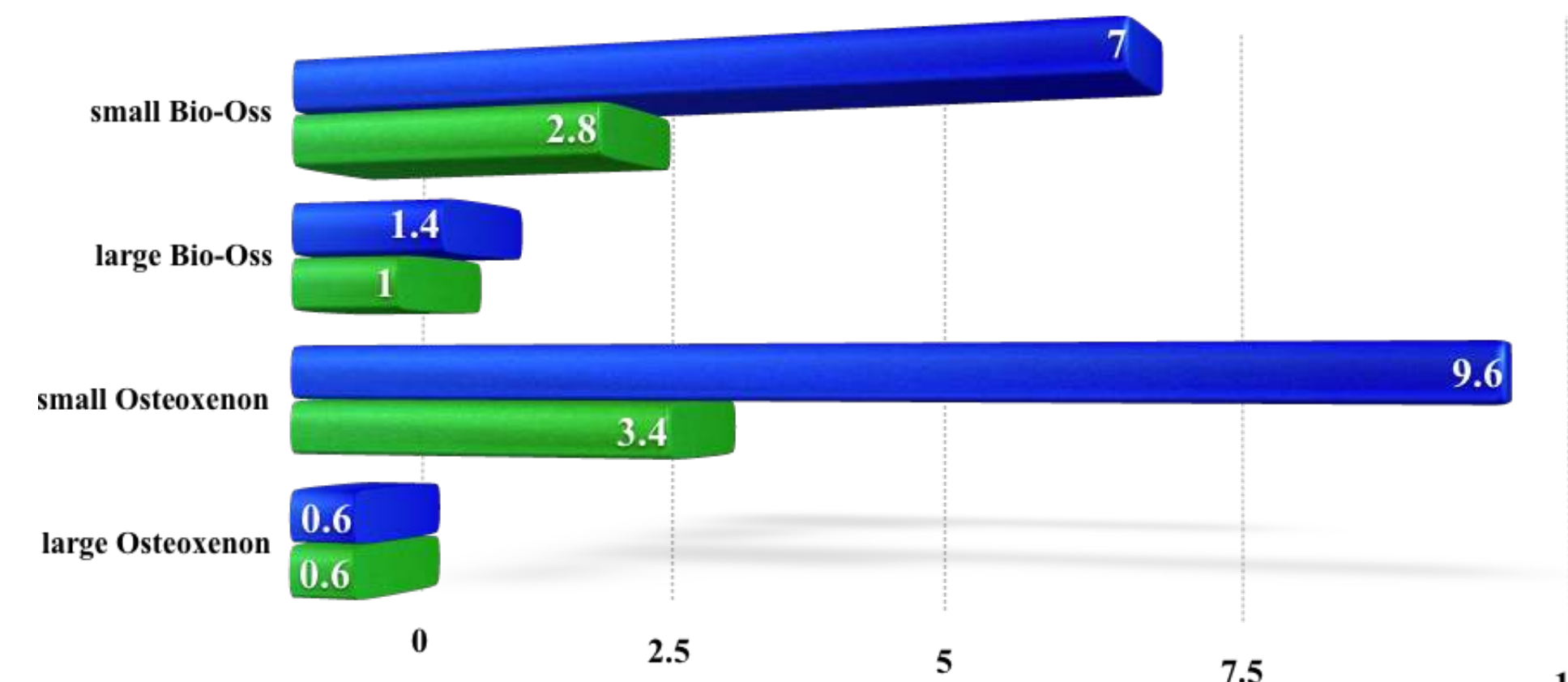

Fig 1 mean value of foreign body giant cells number per $\mathrm{mm}^{2}$

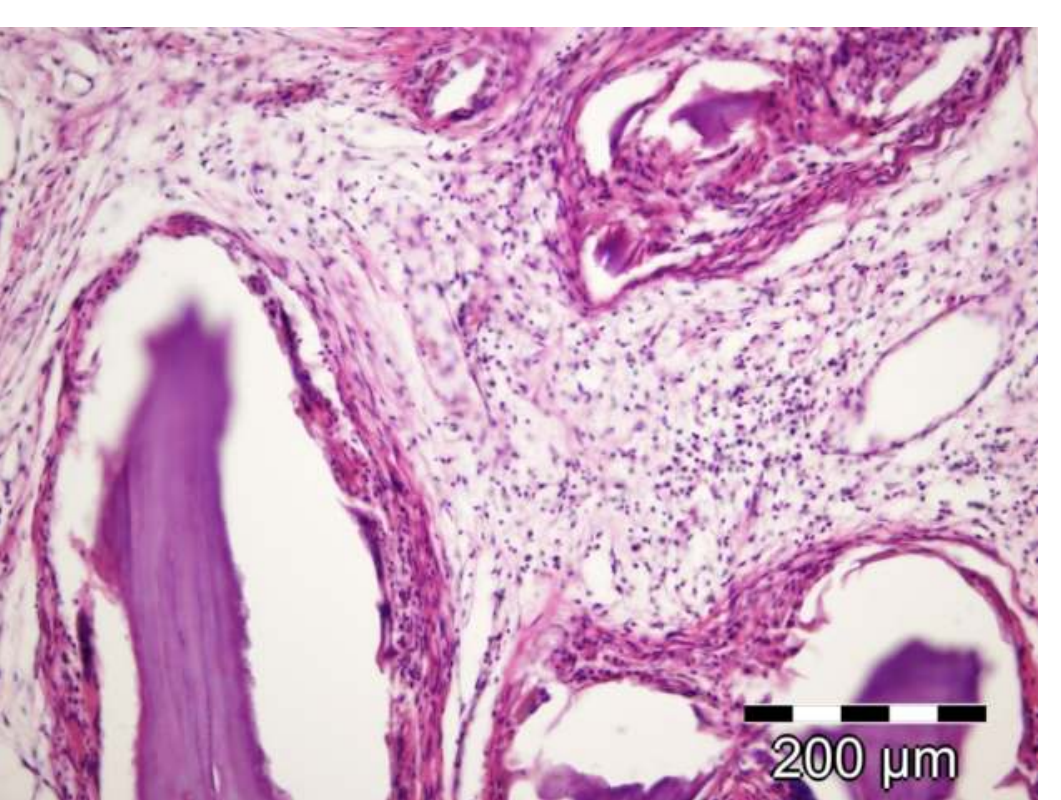

Fig 2 Defect filled with small Osteoxenon particles. Numerous FBGCS surrounding small particles, fibrovascular tissue with numerous mononuclear cells such as
limphocytes and plasmocytes; scarce signs of neoangiogenesis. 4 weeks of healing. (HE $x$
200) 4 weeks
8 weeks

The results showed a larger number of blood vessels in defects filled with large particles after 4 and 8 weeks of healing regardless which xenograft had been used. Considering defects filled with large particles, after 8 weeks of healing the mean value of microvessel density (MVD) was significantly higher in case of large Osteoxenon particles than in large Bio-Oss ones although the difference was numerically small. Regarding to small particles signifficant difference was noticed in favour of those small Bio-Oss (Fig.4).

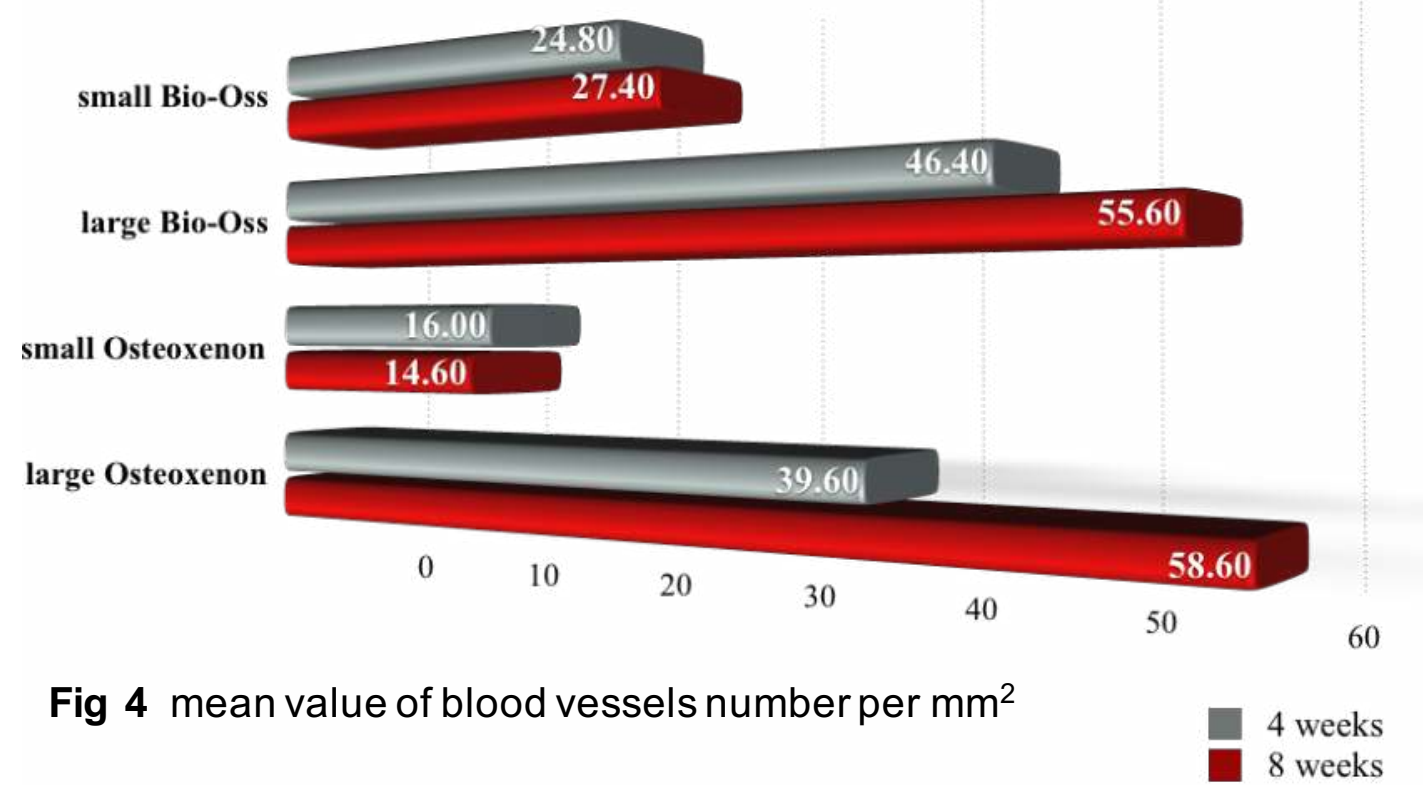

Evaluating fibrovascular tissue after 8 weeks of healing results showed significantly higher rate of fibrovascular tissue production in case of defects filled with large Bio-Oss particles in comparison with all examined substitutes (Fig.5). Taking into consideration the fact that craniofacial sceleton heals by direct intramembranous osification it might be expected that all fibrovascular tissue would be transformed into bone after finishing the regeneration process.

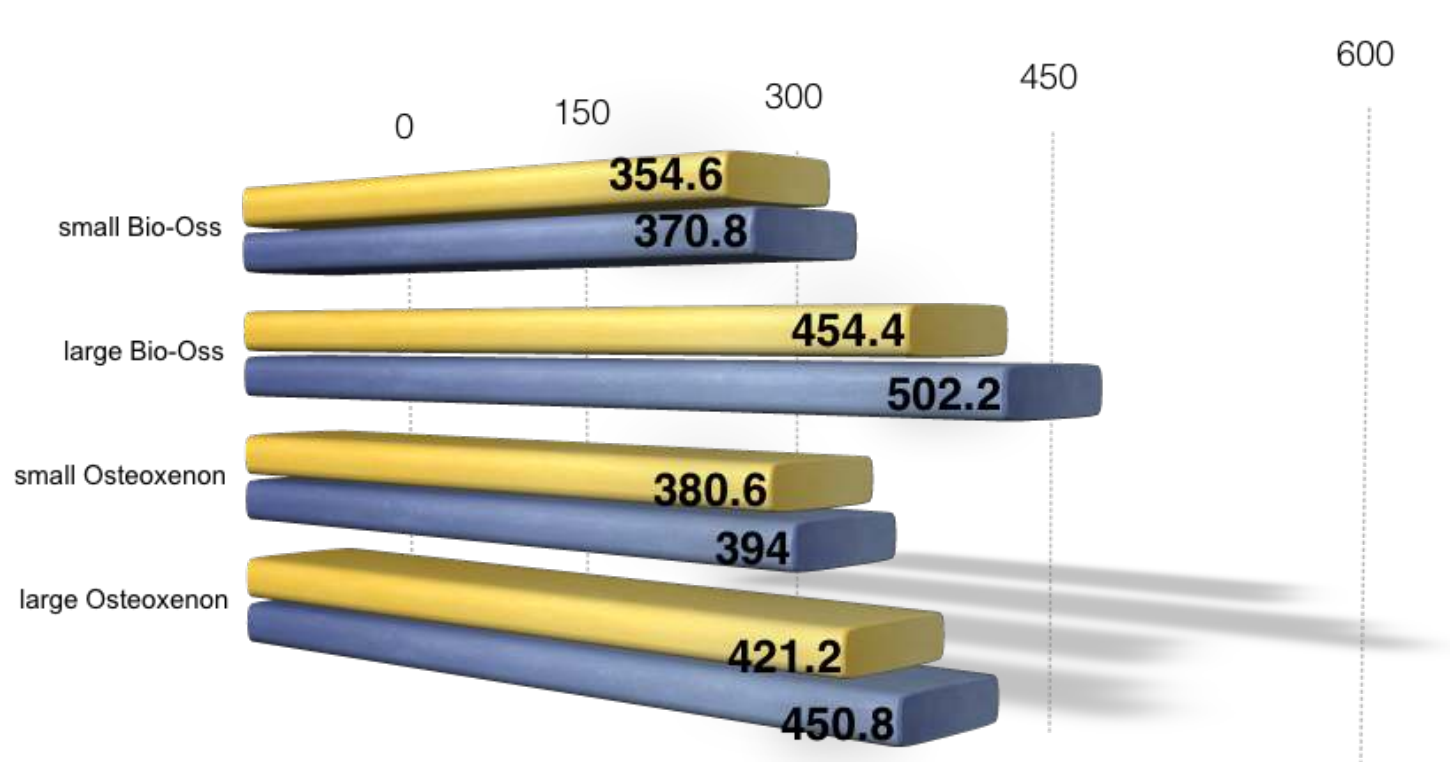

Fig 5 mean value of the highestwidth of fibrovascular strips ( $(\mu \mathrm{m})$

4 weeks
8 weeks

In reference with large particles after 4 and 8 weeks of healing significantly higher bone production was measured in case of large Osteoxenon particles in comparison with Bio-Oss ones.

In terms of new bone production small particles comparison showed difference as well. After 4 weeks of healing it was found significantly greater bone trabecula width in case of defects filled with Bio-Oss than Osteoxenon $(p=0.004)$. However, in case of bone production after 8 weeks of healing significance was lost despite the fact that numerical difference was there (Fig.6).

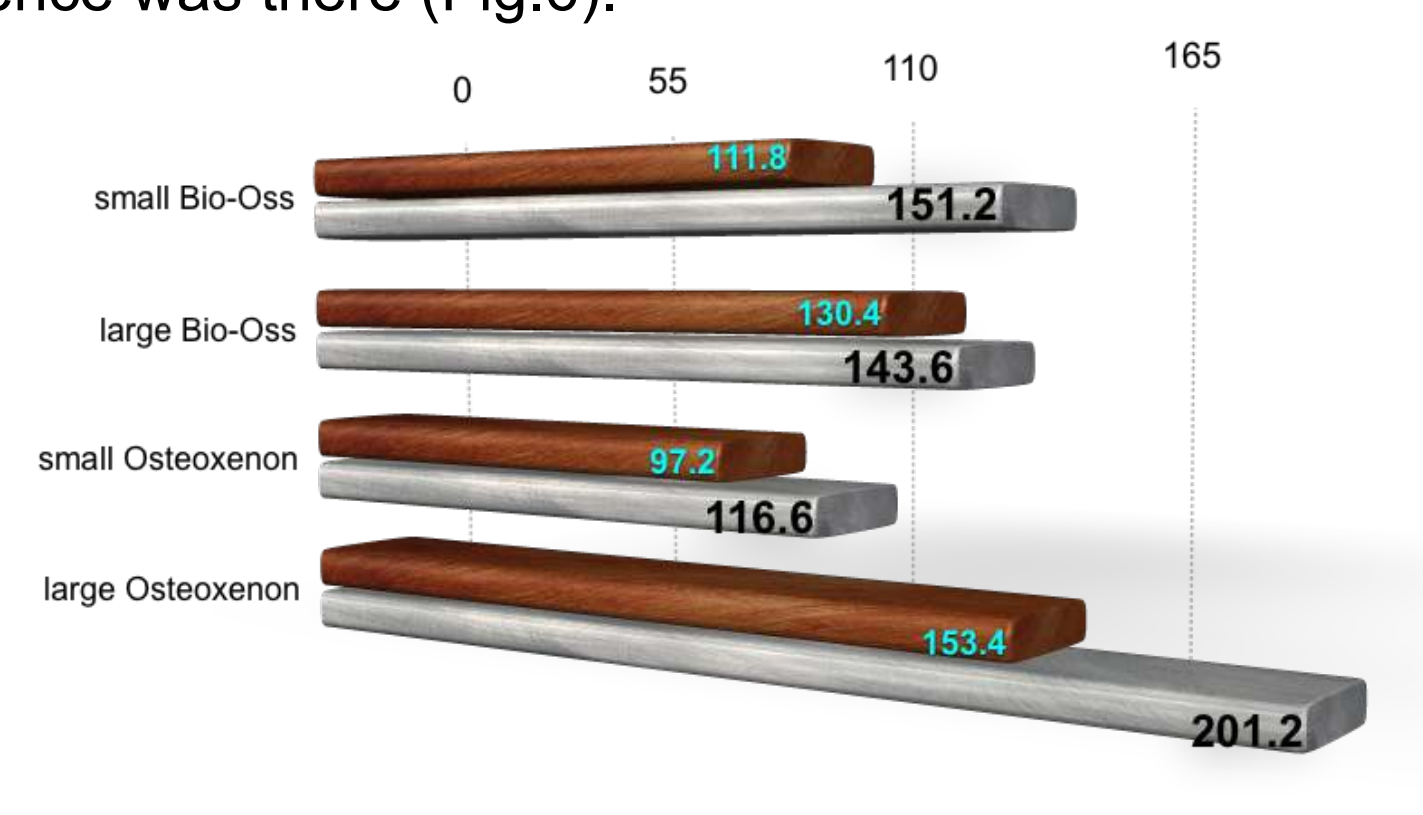

Fig 6 mean value of highestwidth of bone trabeculae $(\mu \mathrm{m})$

\section{4 weeks
8 weeks}

\section{Conclusions}

Within the limits of the present study, it may be concluded:

1. all investigated biomaterials have potential to induce foreign body reaction

2. the size of particles may have greater influence on FBGCs activity than particles porosity

3. small particles induce prolonged non-specific inflammatory reaction associated with foreign body reaction

4. smal particles induce more intense FBGCs reaction than large particles

5. decreasing number of FBGCs taking place during healing process or their sporadic presence around bone substitute particles indicate foreign body reaction as a pattern of successful oseointegration

Fig 3 Defect filled with small Osteoxenon particles. FBGCs in close contact with bone particles; very scant osteoid production; visible pocytes which corresponds to inadequate
bone production. 8 weeks of healing. (HE x 200)
6. procatabolic activity of FBGCs could be associated with less bone production in defects filled with small particles 